\title{
CHARACTERIZATION OF InGaN/GaN-BASED MULTI-QUANTUM WELL DISTRIBUTED FEEDBACK LASERS
}

\author{
Daniel Hofstetter*, Robert L. Thornton**, Linda T. Romano***, David P. Bour***, Michael \\ Kneissl***, Rose M. Donaldson***, and Clarence Dunnrowicz*** \\ *University of Neuchâtel, Institute of Physics, Rue A.-L. Breguet 1, 2000 Neuchâtel, Switzerland \\ Daniel.Hofstetter@iph.unine.ch \\ **MAXTEK Components Corporation, 13335 SW Terman Road, Beaverton, OR 97075-0428 \\ ***XEROX Palo Alto Research Center, 3333 Coyote Hill Road, Palo Alto, CA 94304
}

Cite this article as: MRS Internet J. Nitride Semicond. Res. 4S1, G2.2 (1999)

\begin{abstract}
We present a device fabrication technology and measurement results of both optically pumped and electrically injected InGaN/GaN-based distributed feedback (DFB) lasers operated at room temperature. For the optically pumped DFB laser, we demonstrate a complex coupling scheme for the first time, whereas the electrically injected device is based on normal index coupling. Threshold currents as low as $1.1 \mathrm{~A}$ were observed in $500 \mu \mathrm{m}$ long and $10 \mu \mathrm{m}$ wide devices. The $3^{\text {rd }}$ order grating providing feedback was defined holographically and dry-etched into the upper waveguiding layer by chemically-assisted ion beam etching. Even when operating these lasers considerably above threshold, a spectrally narrow emission $(3.5 \AA)$ at wavelengths around $400 \mathrm{~nm}$ was seen.
\end{abstract}

\section{INTRODUCTION}

Because of their reduced spot size, violet/blue semiconductor lasers are highly advantageous light sources in data scanning and optical storage applications. With the deployment of light emitters fabricated in the InGaN/GaN materials system, the development of an electrically pumped laser emitting light around $400 \mathrm{~nm}$ has experienced tremendous progress during the past couple of years. Under the lead of Nakamura et al., both pulsed and continuous wave operation of InGaN/GaN based devices have been demonstrated at room temperature [1], [2]. One of the main concerns in nitride lasers is the fabrication of high quality mirrors. Dry-etched mirrors with high reflective coatings seem to work pretty well in this material system [3]; however, these etched mirrors are typically not at the edge of the substrate, which complicates the access of the laser beam for most applications. In addition, usually a certain fraction of the optical mode gets diffracted into or reflected from the sapphire surface, leading to an asymmetric far-field with multiple emission lobes in the vertical direction [1], [4]. The use of distributed feedback for providing optical cavity formation could partly eliminate some of the mirror issues because no optical quality mirrors are required to achieve lasing action. Moreover, the longitudinal mode structure, which, due to the very close mode spacing in these devices, is very competitive and noisy, can be markedly improved by using the DFB mechanism for mode selection. A straightforward way to explore this idea is the fabrication of optically pumped DFB lasers [5], [6].

In this article, we present the fabrication of an electrically injected InGaN/GaN-based DFB laser with a holographically defined, index-coupled $3^{\text {rd }}$ order grating. Furthermore, we show some preliminary results on the fabrication of an optically pumped complex-coupled DFB laser. When compared to normal Fabry-Perot devices fabricated from the same material, we observe a 
comparable threshold gain, single mode behavior with a higher side mode suppression ratio, and a modehop-free tuning over a temperature range of more than $30 \mathrm{~K}$.

\section{FABRICATION}

The fabrication of these devices relied on growing a $4 \mu \mathrm{m}$ thick n-type GaN:Si layer on Cface sapphire. On top of this layer, we grew a $500 \mathrm{~nm}$ thick, n-doped $\mathrm{Al}_{0.08} \mathrm{Ga}_{0.92} \mathrm{~N}: \mathrm{Si}$ lower cladding layer, a $100 \mathrm{~nm}$ thick n-doped GaN:Si lower waveguiding layer, a $30 \mathrm{~nm}$ thick un-doped active region with five $3 \mathrm{~nm}$ thick $\operatorname{In}_{0.15} \mathrm{Ga}_{0.85} \mathrm{~N}$ quantum wells and $7 \mathrm{~nm}$ thick GaN barriers, and a $180 \mathrm{~nm}$ thick p-doped GaN:Mg upper waveguiding layer. For the electrically injected, indexcoupled device, the $3^{\text {rd }}$ order grating with a period of $240 \mathrm{~nm}$ was defined by a holographic exposure and dry-etched into the upper waveguiding layer by chemically-assisted ion beam etching. A numerical calculation of the coupling coefficient showed that the tooth shape of this $100 \mathrm{~nm}$ deep grating was a critical parameter. For our rectangular tooth geometry with rounded tops, the coupling coefficient was relatively weak, on the order of $5-10 \mathrm{~cm}^{-1}$. Together with a cavity length of $1000 \mu \mathrm{m}$, this coupling strength corresponds to an effective reflectance of 15 $30 \%$. After grating fabrication, we performed optical pumping experiments in order to confirm the matching between the grating resonance wavelength and the gain peak. Then, we proceeded with an epitaxial re-growth to complete the device structure. This re-growth consisted of a $300 \mathrm{~nm}$ thick p-doped $\mathrm{Al}_{0.08} \mathrm{Ga}_{0.92} \mathrm{~N}: \mathrm{Mg}$ upper cladding layer and a $100 \mathrm{~nm}$ thick p-type $\mathrm{GaN}: \mathrm{Mg}$ contact layer.

Device processing for the electrically pumped devices involved the definition mesas to enable lateral n-type contacting of the devices, and evaporation of standard n- and p-metal Ti/Au layers. The p-metal contact stripes were 4,10 or $20 \mu \mathrm{m}$ wide, and thus defined the width of the gain region. A SiON layer was used to electrically isolate the non-contacted areas and the

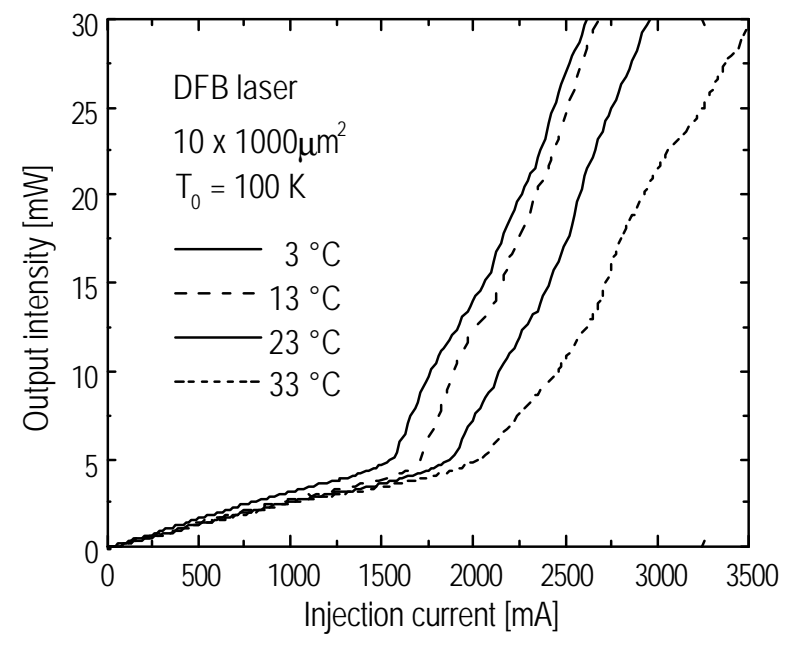

Fig. 1 - L-I-characteristics of an InGaN / GaN DFB laser with an active area of $10 x$ $1000 \mu^{2}$ at four different heatsink temperatures. sidewalls of the mesas, thereby restricting gain to within a narrow stripe in the lateral direction. Following the SiON window etch, we evaporated another $\mathrm{Ti} / \mathrm{Au}$ layer in order to provide the p-metal contact pads for the probe. Finally, the mirrors were etched, again by chemically-assisted ion beam etching. In order to ensure that these lasers would not accidentally oscillate as FabryPerot lasers, we dry-etched only one of the facets exactly vertical while leaving the other one at an angle of approximately $20^{\circ}$ to the vertical.

The fabrication of optically pumped complex-coupled DFB lasers relied on interrupting growth after the QW active region, and etching the grating with a depth of $45 \mathrm{~nm}$ through the QWs. Re-growth of $100 \mathrm{~nm} \quad \mathrm{GaN}$ and a $50 \mathrm{~nm}$ thick $\mathrm{Al}_{0.08} \mathrm{Ga}_{0.92} \mathrm{~N}: \mathrm{Mg}$ layer took place to complete the optical waveguide and a small fraction of the upper cladding layer. Because of the 
resulting gain and refractive index variation, this coupling scheme should be much stronger than index coupling alone. Optically pumped lasers could then be fabricated by simply cleaving $6 \mathrm{~mm}$ wide and $10 \mathrm{~mm}$ long pieces.

\section{ELECTRICALLY INJECTED DFB LASER}

Measurements of the I-V- and L-I-characteristics were carried out under pulsed current conditions (500 ns pulse length, $0.05 \%$ duty cycle) at room temperature. The lasers were probe contacted and tested on chip. At $1 \mathrm{~mA}$ forward current, the I-V-curve typically exhibited a "turnon voltage" of $3.3 \mathrm{~V}$, and for a $10 \times 1000 \mu \mathrm{m}^{2}$ contact region, we observed a series resistance of $10 \Omega$. As shown in the L-I-curves of figure 1 , we obtained, for the longest devices with 10 microns stripe width, pulsed threshold currents of $1550 \mathrm{~mA}$ at $3{ }^{\circ} \mathrm{C}$ and $2050 \mathrm{~mA}$ at $33{ }^{\circ} \mathrm{C}$. From these measurements, we determined the $\mathrm{T}_{0}$ value to be approximately $100 \mathrm{~K}$.

The best threshold currents were obtained on lasers with 20 microns stripe width and $1000 \mu \mathrm{m}$ cavity length. Values of $3200 \mathrm{~mA}$ for 20 microns wide devices were observed, corresponding to threshold current densities of $16 \mathrm{kA} / \mathrm{cm}^{2}$. For shorter devices with 20 microns stripe width, we measured higher threshold currents of $4600 \mathrm{~mA}$ (threshold current density of $\left.23 \mathrm{kA} / \mathrm{cm}^{2}\right)$. For the best devices, typical threshold voltages of $\mathrm{V}\left(\mathrm{I}_{\mathrm{th}}\right)=16 \mathrm{~V}$ were seen.

High-resolution spectra of these lasers were measured using a grating spectrometer with a resolution of $0.5 \AA$ (SPEX, $\left.\mathrm{d}_{\text {focal }}=1.26 \mathrm{~m}, \mathrm{w}_{\text {slit }}=50 \mu \mathrm{m}\right)$. The laser output was focussed onto a quartz fiber using a microscope objective, and fed into the input slit of the spectrometer. By placing a 1024 element array photodetector into the output image plane, we were able to measure the spectrum in a spectral window of $10 \mathrm{~nm}$ width within 1 second.

In figure 2, we show high-resolution emission spectra at various current levels ranging from $1.01 \mathrm{x} \mathrm{I}_{\text {th }}$ and to $1.28 \times \mathrm{I}_{\text {th. }}$. While there is only one clean peak with a resolution limited width of $0.5 \AA$ for lower injection currents, there appear to be multiple peaks with an overall width of $5 \AA$ at higher injection levels. The fact that all additional features occurred at the long wavelength side of the main peak suggests that chirping due to device heating during the current pulse is responsible for this kind of broadening. From the spectral broadening at higher injection currents and the temperature tuning coefficient derived in the following paragraph, we were able to estimate the temperature increase during the pulse to be on the order of $20-30 \mathrm{~K}$. Another possible reason for the spectral broadening at higher current levels is the onset of lasing in higher order lateral modes in these gain-guided structures. This explanation is supported by the fact that the L-I-curves show several kinks.

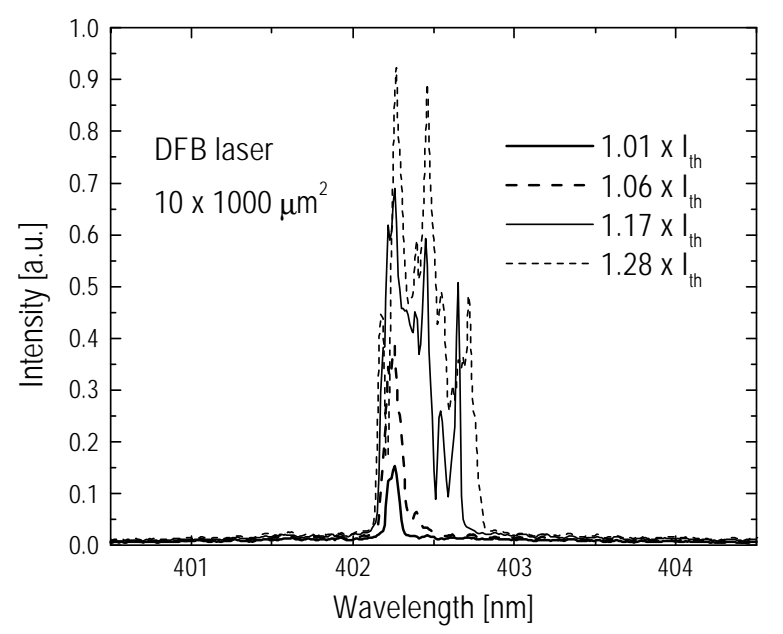

Fig. 2 - Emission spectra of an InGaN/GaN DFB laser at four different injection current levels ranging from $1.01 \times I_{\text {th }}$ to $1.28 \times I_{\text {th }}$. All curves are drawn using the same vertical scale. 
Emission spectra of a $1000 \mu \mathrm{m}$ long DFB laser at four different heat-sink temperatures are shown in figure 3; they reveal laser oscillation in a single longitudinal mode with a sidemode suppression ratio of $15 \mathrm{~dB}$ and at a center wavelength of around $402 \mathrm{~nm}$. Given the grating period of $240 \mathrm{~nm}$ and the above emission wavelength, we were able to calculate the effective refractive index of the propagating mode to be $n_{\text {eff }}=2.52$. In order to maintain narrow emission spectra for this measurement, we adjusted the injection current to always be at $1.1 \mathrm{x}_{\mathrm{th}}$. The device remained in a single mode for temperatures ranging from $2{ }^{\circ} \mathrm{C}$ to $35^{\circ} \mathrm{C}$, which is a temperature range of more than $30 \mathrm{~K}$; the temperature tuning coefficient was on the order of $0.014 \mathrm{~nm} / \mathrm{K}$. For FabryPerot type lasers fabricated on the same wafer, we measured a much larger average temperature tuning coefficient of $0.065 \mathrm{~nm} / \mathrm{K}$.

\section{OPTICALLY PUMPED COMPLEX COUPLED DFB LASER}

Cross-sectional transmission electron microscope (TEM) studies of both types of overgrowth reported in this article showed that no additional dislocations were created at the grating interface. This observation is in sharp contrast to InGaAsP/InP-based infrared DFB lasers, where the grooved interface serves as starting point for numerous dislocations [7]. However, we found also that only 3 out of 5 QWs were etched during the grating etch process; this conclusion confirmed that our device was a complex-coupled rather than a purely gain-coupled DFB laser. The reason for the insufficient etch-depth was mainly the lack of process control when trying to punch through all QWs without over-etching. From a calculation of the coupling strength for this complex-coupled sample, we estimated similar amounts of index- and gain-coupling ( $\kappa=5$ $10 \mathrm{~cm}^{-1}$ each).

Optical pumping was carried out as described earlier using a pulsed $337 \mathrm{~nm} \mathrm{~N}$ laser $\left(\mathrm{r}_{\text {pulse }}=\right.$

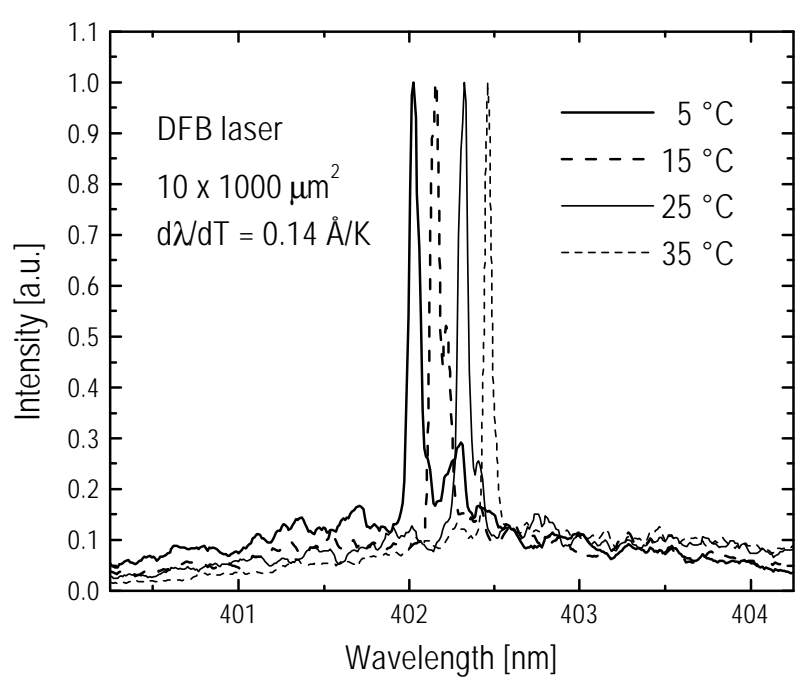

Fig. 3 - Emission spectra of the same device as in figure 2 at $1.1 \times I_{\text {th }}$ and at different heat-sink temperatures. The temperature tuning coefficient obtained was $0.14 \AA / K$.
$10 \mathrm{~Hz}, \mathrm{P}_{\text {peak }}=250 \mathrm{~kW}$ ) whose stripeshaped beam was attenuated by an appropriate number of glass slides. The output of the InGaN/GaN laser was fed into either a high resolution spectrometer (for linewidth measurements) or a low resolution grating spectrometer (for output intensity vs. current-curves). The pieces on which we performed these experiments were approximately $6 \mathrm{x}$ $10 \mathrm{~mm}^{2}$ in size, and all four facets were fabricated by scribing from the back and subsequent cleaving. Although this method resulted in a poor Fabry-Perot (FP) cavity and, in addition, the $\mathrm{N}_{2}$ laser beam was pumping $30-40 \%$ of the cavity length only, FP type laser emission could be observed as well as DFB laser emission. The orientation of the grating lines relative to the pump beam determined which emission would 


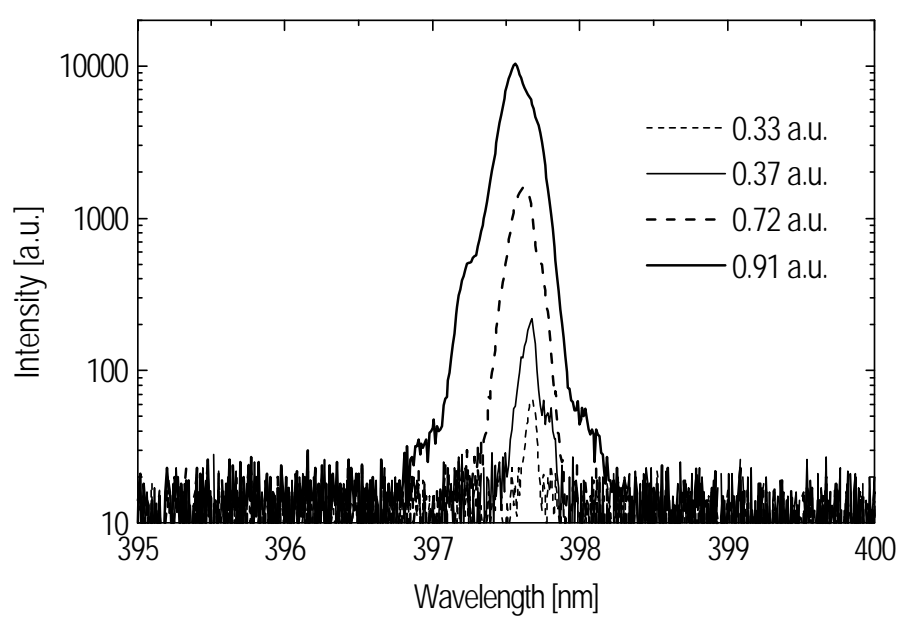

Fig. 4 - Emission spectra of an optically pumped complex-coupled DFB laser at different pump levels become the dominant one.

Typical emission spectra of DFB type lasing are shown in figure 4 . The emission peak stays at $397.5 \mathrm{~nm}$ from threshold all the way up to 0.9 a.u. pump intensity. It shifts somewhat towards shorter wavelengths at higher pump intensities. The reason for this wavelength shift is a slight reduction of the effective refractive index at high carrier density, which, at some point, overwhelms the index increase due to the relatively weak heating effects. The linewidth change from $1.5 \AA$ right above threshold to $3.5 \AA$ at 0.91 a.u. is likely due to lateral multimode operation and some chirping at high

pump intensities. A similar series of low resolution emission spectra for the FP emission showed a peak wavelength of $395 \mathrm{~nm}$ with a typical linewidth of around $25 \AA$. In contrast to the DFB laser spectra, we see for the FP laser a pronounced red shift of the lasing peak when pumping at a higher intensity. While the DFB lasing wavelength is locked to the Bragg resonance wavelength, the emission of the FP follows the gain maximum, which, again due to device heating, shifts more rapidly towards longer wavelengths.

The far field of the DFB lasers reported in this article exhibited the usual interference effects due to the reflection from and the diffraction into the sapphire substrate. However, in the lateral direction, we observed a far field angle of approximately $10^{\circ}$ above threshold, and a broad Lambertian characteristics below threshold. On Fabry-Perot lasers from similar material, we polished one mirror facet in order to get a far field measurement without the diffraction and refraction artifacts. Due to the insufficiently thick AlGaN lower cladding layer, the result of this measurement was very similar to what we reported earlier on optically pumped lasers [4]. It revealed two main emission lobes in the vertical direction and a number of weaker oscillations in between.

\section{CONCLUSIONS}

In conclusion, we have demonstrated electrically injected index-coupled and optically pumped complex-coupled InGaN/GaN-based DFB lasers. The best threshold current density observed was comparable to that of conventional Fabry-Perot type devices, and was on the order of $16 \mathrm{kA} / \mathrm{cm}^{2}$. The $\mathrm{T}_{0}$ value describing the $\mathrm{T}$-dependence of the threshold current was approximately $100 \mathrm{~K}$. The reason for the much better $\mathrm{T}_{0}$ value measured on Fabry-Perot devices fabricated from the same material $\left(\mathrm{T}_{0}=150 \mathrm{~K}\right)$ is the increasing mismatch between the grating resonance wavelength and the gain maximum when heating or cooling the DFB lasers. The emission of the DFB laser was at a wavelength of $402 \mathrm{~nm}$ and occurred in a single longitudinal mode with a sidemode suppression ratio of $15 \mathrm{~dB}$. The primary emission peak could be 
temperature tuned continuously between 2 and $35^{\circ} \mathrm{C}$ at a rate of $0.14 \AA / \mathrm{K}$. The optically pumped device showed a narrow linewidth of $3.5 \AA$, even for pump intensities considerably above threshold, and almost no wavelength shift at higher pump intensities.

\section{ACKNOWLEDGEMENTS}

The authors would like to thank Fred Endicott and Greg Anderson for technical assistance, and Noble M. Johnson, Ross Bringans, Gary A. Evans (Southern Methodist University, Dallas, TX), and Peter S. Zory (University of Florida, Gainesville, FL) for helpful discussions. This work was supported by the Defense Advanced Research Projects Agency (DARPA contract number MDA 972-96-3-0014), and the Swiss National Science Foundation.

\section{REFERENCES}

[1] S. Nakamura, M. Senoh, S. Nagahama, N. Iwasa, T. Yamada, T. Matsushita, Y. Sugimoto, and H. Kiyoku, InGaN-based multi-quantum-well structure-laser diodes, Jpn. J. Appl. Phys., 35 (1), L74 - L76 (1996)

[2] D.P. Bour, H.F. Chung, W. Götz, B.S. Krusor, D. Hofstetter, S. Rudaz, C.P. Kuo, F.A. Ponce, M.G. Craford, and R.D. Bringans in III-V Nitrides, edited by T.D. Moustakas, I. Akasaki, B. Monemar, and F.A. Ponce (Mater. Res. Soc. Proc. 443, Pittsburgh, PA, 1996) pp. $509-514$

[3] M. Kneissl, D.P. Bour, N.M. Johnson, L.T. Romano, B.S. Krusor, R. Donaldson, J. Walker, and C.Dunnrowicz, Characterization of AlGaInN diode lasers with mirrors from chemically assisted ion beam etching, Appl. Phys. Lett., 72 (13), 1539 - 1541 (1998)

[4] D. Hofstetter, D.P. Bour, R.L. Thornton, and N.M. Johnson, Excitation of a higher order transverse mode in an optically pumped $\mathrm{In}_{0.05} \mathrm{Ga}_{0.95} \mathrm{~N} / \mathrm{In}_{0.15} \mathrm{Ga}_{0.85} \mathrm{~N}$ multiquantum well laser structure, Appl. Phys. Lett., 70 (13), 1650 - 1652 (1997)

[5] D. Hofstetter, M. Kneissl, D.P. Bour, C. Dunnrowicz, and R.L. Thornton, Demonstration of an InGaN/GaN-based optically pumped multi quantum well DFB laser using holographically-defined $3^{\text {rd }}$ order gratings, Appl. Phys. Lett., 73 (14), 1918 - 1920 (1998)

[6] R. Hofmann, H.-P. Gauggel, U.A. Griesinger, H. Gräbeldinger, F. Adler, P. Ernst, H. Bolay, V. Härle, F. Scholz, H. Schweizer, and M.H. Pilkuhn, Realization of optically pumped second-order GaInN-distributed-feedback lasers, Appl. Phys. Lett., 69 (14), 2068 - 2070 (1996)

[7] S.N.G. Chu, T. Tanbun-Ek, R.A. Logan, J. Vandenberg, P.F. Sciortino, Jr., P. Wisk, and T.L. Pernell, Grating overgrowth and defect structures in distributed-feedback-buried hererostructure laser diodes, IEEE Journal of Selected Topics in Quantum Electronics, 3 (3), $862-873$ (1997) 